\title{
The urokinase-system in tumor tissue stroma of the breast and breast cancer cell invasion
}

\author{
RALF HILDENBRAND ${ }^{1,2}$ and ANTONELA SCHAAF ${ }^{1}$ \\ ${ }^{1}$ Institute of Pathology, Medical Faculty Mannheim, University of Heidelberg, Theodor-Kutzer-Ufer 1-3, \\ 68167 Mannheim; ${ }^{2}$ Institute of Pathology, Heilsbachstrasse 15, 53123 Bonn, Germany
}

Received August 13, 2008; Accepted October 10, 2008

DOI: 10.3892/ijo_00000124

\begin{abstract}
The urokinase-system has been implicated in tumor spread. The serine protease urokinase-type plasminogen activator (UPA), its receptor (UPAR) and its inhibitor (PAI-1) are involved in the control of extracellular turnover, cell migration, invasion, cell signalling, proliferation, apoptosis and angiogenesis leading to a variety of different responses, under both physiological and pathological conditions. UPA and PAI-1 were the first novel tumor biological factors to be validated at the highest level of evidence regarding their clinical utility in breast cancer. However, it is unclear whether it is their (relative) levels in the tumor stroma or in the tumor cells themselves that is the most relevant to patients outcome. This is the first study in which tumor cells and stromal tissue of invasive breast carcinomas were separated by laser capture microdissection followed by ELISA-based determination of the UPA, uPAR and PAI-1 levels. In addition, we localized uPA, uPAR and PAI-1 distribution in invasive breast cancer $(\mathrm{n}=30)$ and in ductal carcinoma in situ (DCIS, $\mathrm{n}=30)$ by immunohistochemistry and in situ hybridization. We have demonstrated that no significant differences between uPA, UPAR and PAI-1 levels in tumor stroma only, tumor cells only and not separated breast cancer tissue exist ( $\mathrm{p}>0.05)$. Our results suggest that similar expression levels of these factors in both compartments and in not separated breast cancer tissue may have the same impact on the clinical behavior of breast cancer. These results are an important issue for practical use of tissue sampling. For using uPA and PAI-1 levels as prognostic and predictive factors in breast cancer the quantity of tumor stroma in the tumor tissue specimen is not relevant for assessment patients outcome. Our results were confirmed by immunohistochemistry and in situ hybridization analysis showing that in nearly all cases of invasive carcinomas and
\end{abstract}

Correspondence to: Dr Ralf Hildenbrand, Institut für Pathologie, Heilsbachstrasse 15, 53123 Bonn, Germany

E-mail: hildenbrand@patho-bonn.de

Key words: urokinase-type plasminogen activator, plasminogen activator inhibitor-1, urokinase-type plasminogen activator receptor, breast cancer, ductal carcinoma in situ, tumor stroma, myoepithelial cells
DCIS fibroblasts as well as macrophages strongly express uPA, uPAR and PAI-1. Prompted by our immunohistological results that nearly all myoepithelial cells of DCIS exhibit uPA, UPAR and PAI-1, we investigated these important host cells in detail. We have demonstrated by multimodal methods that UPAR and PAI-1 protein and mRNA is expressed in most myoepithelial cells of DCIS. Additionally, we furnish evidence that UPAR expression of myoepithelial cells are important for uPAR Vitronectin-associated cell-matrix interaction, which regulates cell adhesion and detachment. We speculate that the loss of the anti-invasive myoepithelial cell layer in DCIS may be triggered by PAI-1 and could be an early sign of subsequent tumor cell invasion.

\section{Introduction}

The stroma is essential for the maintenance of epithelial tissue. When the epithelium changes, the stroma inevitably changes also. This is the case during cancer development although momentum concerns the oncogenic alterations in the epithelial cells. Genetically changed epithelial cells (cancer cells) change the stromal host compartment so as to establish a permissive and supportive environment, making cancer an alien entity invading the body. Clinical and experimental data support the notion that host factors may regulate invasion and metastasis (1).

In previous decades, evidence has been accumulating showing the important role of the urokinase-system in growth, invasion and metastasis. UPA and PAI-1 were the first novel tumor biological factors to be validated at the highest level of evidence regarding their clinical utility in breast cancer. The serine protease urokinase-type plasminogen activator (uPA), its receptor (uPAR) and its inhibitor (PAI-1) are involved in the control of extracellular turnover, cell migration, invasion, cell signalling, proliferation, apoptosis and angiogenesis leading to a variety of different responses, under both physiological and pathological conditions (2-7). However, it is unclear whether it is their (relative) levels in the tumor stroma/host bystander cells or in the tumor cells themselves that is most relevant to patients outcome. It has become clear that cancer cells come under the influence of important paracrine regulation from the host microenvironment. Such host regulation may be as great a determinant of tumor cell behavior in vivo as the specific oncogenic or tumor suppressor alterations occurring within the malignant cells themselves and 
may be mediated by specific extracellular matrix molecules, matrix-associated growth factors, or host cells themselves (8). Both positive and negative cellular regulators (macrophages, fibroblasts/myofibroblasts, lymphocytes, endothelial cells, myoepithelial cells) exist that profoundly affect tumor cell behavior in vivo (9).

The role of the urokinase-system in the tumor stroma and host bystander cells is poorly understood. For determination and localization of components of the uPA-system in the stroma and host bystander cells we performed immunohistochemistry-based and ELISA-based studies. This is the first study in which tumor cells and stromal tissue of invasive breast carcinomas were separated by laser capture microdissection followed by ELISA-based determination of the UPA, uPAR and PAI-1 levels. Furthermore, we analyzed the components of the uPA-system in precursor lesions of the breast cancer (DCIS) and normal breast tissue by immunohistochemistry and in situ hybridization. Prompted by our immunohistological results that nearly all myoepithelial cells exhibit uPA, uPAR and PAI-1, we investigated these important host cells in detail.

\section{Materials and methods}

Patients and histomorphological factors. Thirty patients with invasive breast cancer $(n=30)$ and 30 patients $(n=30)$ with ductal carcinoma in situ (DCIS) were enrolled in this study. Primary treatment was by modified radical mastectomy or by breast conserving therapy including axillary lymph node dissection or sentinel node biopsy in cases of invasive cancer. None of the patients received any neoadjuvant therapy. The median number of axillary lymph nodes removed in cases of invasive breast cancer was 15 (range 10-20). Eighteen patients were node negative and in 12 patients the lymph nodes were infiltrated by tumor cells. The DCIS patients were on average $54.5 \pm 1.4$ years of age and the patients with invasive breast cancer were on average $57.4 \pm 1.5$ years of ages. The size of the invasive tumors ranged from 12 to $66 \mathrm{~mm}$. The size of the DCIS ranged from 15 to $70 \mathrm{~mm}$. All tumor sections were stained with haematoxylin and eosin and the invasive carcinomas were graded according to the Elston and Ellis criteria (10). Eight tumors were grade 1, 14 were grade 2 and 8 tumors were grade 3 . The DCIS were graded according to the WHO: 9 cases were low grade, 12 cases were intermediate grade and 9 cases were high grade.

Immunohistochemistry and double immunostaining. Immunohistochemistry was performed on $2-\mu \mathrm{m}$ thick paraffinsections using a mouse anti-uPAR monoclonal antibody (clone 3B10, IgG2a; American Diagnostica \#3936; 1:100 dilution) to uPAR expressed by phorbol ester-stimulated promyeloid U937 cells, mAb IID7 to human nonglycosylated uPAR polypeptide ${ }_{1-284}$ expressed in E. coli (11) and chicken polyclonal antibody ( $\operatorname{IgY})$ directed to affinity-purified recombinant human $\mathrm{uPAR}_{1-277}$ expressed in Chinese hamster ovary $(\mathrm{CHO})$ cells reacting with native and denaturated cellassociated uPAR antigen (12-14) (pAb HU277; kindly provided by Viktor Magdolen and Manfred Schmitt; Klinische Forschergruppe der Frauenklinik, TU-München, Germany; 1:100 dilution). Consecutive tissue sections were stained with
mAbs to anti-uPA (American Diagnostica, Germany, \#3688) and anti-PAI-1 (American Diagnostica, Germany \#3785). Immunostaining was based on an alkaline phosphataseconjugated streptavidin-biotin detection system (Amersham Pharmacia Biotech Inc., Piscataway, NJ), using Fast Red (Roche Diagnostics $\mathrm{GmbH}$ ) as a chromogen. For both antibodies antigen retrieval was achieved by microwave treatment $(3 \times 5 \mathrm{~min}, 600 \mathrm{~W})$. Incubation of the primary antibody was carried out for $60 \mathrm{~min}$ at $37^{\circ} \mathrm{C}$. Negative controls were performed by substituting non-immune IgG. Additionally the staining reaction was blocked by preincubation of $\mathrm{pAb}$ HU277 with an excess of the CHO-uPAR ${ }_{1-277}$ prior to the staining reaction. For recognising macrophages, endothelial cells, fibroblasts, myofibroblasts, smooth muscle cells and lymphocytes, consecutive tissue sections were stained with $\mathrm{mAb}$ to CD34, CD68, CD45, smooth muscle antigen (SMA) and to fibroblast antigen (all from Dako, Hamburg, Germany). The anti-ER (clone 1D5), anti-PgR (clone PgR636) and antiKi-67 (clone MIB-I) antibodies (all mouse monoclonal) as well as Hercep Test system for the detection of the HER2/neu expression came from Dako. Immunohistochemistry was performed using a standard biotin-streptavidin method with the appropriate antigen retrieval method for each antibody. With execption of Hercep Test, we used Fast Red (Roche, Mannheim, Germany) as chromogen. Nuclei were counterstained with hematoxylin.

In DCIS cases a double staining was performed starting with the mAb IID7 (mAb anti-PAI-1) detected with the APAAP-method then proceeding with $\mathrm{mAb}$ anti-calponin detected with the streptavidin-biotin-POD-method. For the second reaction the DAB staining kit (Leinco, k107, Germany) (diaminobenzidin) and 'metal enhancing solution' was used. The immunostaining is in red (APAAP, neufuchsin) and in black (streptavidin-biotin-peroxidase, DAB plus metalenhancing solution) and nuclei were conterstained with hematoxylin (blue color). In each case negative controls were performed by substituting non-immune-antibodies ( $\mathrm{IgG}$ ) for mAb \#3936, pAb HU277 and mAb IID7, respectively. Additionally, the staining reaction was blocked by preincubation of pAb HU277 with an excess of CHO-uPAR ${ }_{1-277}$ prior to the staining reaction.

In 10 cases of DCIS a collagen type-4/vitronectin doublestaining was performed. The sections were incubated with $\mathrm{mAb}$ anti-vitronectin (Innovex Bioscience, Germany; dilution 1:50), washed and incubated with Texas Red sulfonyl chlorid (TRSC)-conjugated rabbit anti-mouse IgG (Dianova, Hamburg, Germany, ditution 1:1000). Sections were then incubated with anti-collagen-type-4 (Dako; $100 \mu 1 /$ section) that had been biotinylated previously (ARK biotinylating kit; Dako). The biotin label was subsequently visualized with FITC-conjugated streptavidin (Vector Laboratories, Burlingame USA; dilution 1:250). Controls were incubated with non-immune antibodies applied at the concentration as the primary mAb. In the controls, no specific immunolabeling was observed.

In situ hybridization. In situ hybidization with fluoresceinlabeled oligodeoxynucleotides was performed following the protocol of Hildenbrand and co-workers. For detection of fluorescein-labeled oligodeoxynucleotides, the 'Super Sensitive 
mRNA Probe Detection System' (BioGenex, CA, USA) was used. The antisense oligodeoxynucleotides (Biometra, Germany) were complementary to nucleotides 121-150, 321$350,521-550,717-746$ and 918-947 of uPAR mRNA and 181-210, 421-450, 661-690, 901-930 and 1081-1110 of PAI-1 mRNA (according to the nucleotide numbering of accesion number X51675 in the EMBL data base). For mRNA detection in invasive breast cancer tissues we have used a black and in cases of DCIS a red color solution $(13,14)$.

Laser capture microdissection for separating tumor stroma and cancer cells. Serial-frozen sections $(4-8 \mu \mathrm{m})$ were cut on a standard cryostat (Leica, Germany) with a clean blade. The unfixed tissue sections were immediately stored at $-80^{\circ} \mathrm{C}$ until use. The frozen sections were stained by toluidine blue (minimal amount of staining to visualize the tissue for microdissection; \#19.816-1; Sigma, Germany). After staining and microscopic control of staining quality and tissue preservation, microdissection was performed using a laser capture microdissection microscope (Arcturus) equipped with an infrared laser. The tissue sections were overlaid with optically transparent caps and tissue/cells were captured by focal melting through laser activation. After visual control of the completeness of dissection, captured tissue/cells were washed TBS (0.002 M Tris HCl, 0.125 M NaCl, pH 8.5) and destained. Carcinoma cells were completely separated from tumor stroma by microdissection yielding 250-300 mg wet weight of separated carcinoma cells and tumor cell-free stromal tissue. Unprocessed (not microdissected) frozen tumor sections were treated (washed and destained) equally yielding also 250-300 mg wet weight (tumor cells and tumor stroma). Between 40 and 80 tissue sections were microdissected for receiving 250-300 $\mathrm{mg}$ wet weight per case.

Laser capture microdissection of immunostained frozen sections for mRNA analysis. The frozen sections were thawed at room temperature for 30-60 sec and immersed immediately in cold acetone $(5 \mathrm{~min})$. After fixation, the slides were rinsed briefly in phosphate-buffered saline (PBS, pH 7.4) and subjected to immunostaining. The immunostaining was performed with a modified Dako staining kit (Dako), a three-step streptavidin-biotin technique with prediluted monoclonal anti-smooth muscle actin antibodies (anticalponin, 1:80, BioGenex, Germany; and anti- $\alpha$-smooth muscle actin, 1:100, Dako) optimized for very short staining times. The slides were incubated at room temperature with the primary and secondary antibodies and an alkalinephosphatase-conjugated antibody for 90-120 sec each and briefly rinsed in PBS between each step. After color development with $0.04 \%$ 5-bromo-4-chloro-3-indolylphosphate (Roche, Mannheim, Germany) and $0.06 \%$ nitro blue tetrazolium (Sigma, München Germany) for 3-5 min and counterstaining with hematoxylin for $20 \mathrm{sec}$, the sections were dehydrated in graded alcohols (15 sec each) and xylene ( $2 \times 2 \mathrm{~min})$ and air dried. After immunostaining and microscopic control of staining quality and tissue preservation, microdissection was performed using a laser capture microdissection microscope (Arcturus) equipped with an infrared laser. The dehydrated tissue section is overlaid with optically transparent caps and cells were captured by focal melting through laser activation. After visual control of the completeness of dissection, the captured cells (myoepithelial cells) were immersed in denaturation solution (15).

Tissue extraction and ELISA. Tissue extraction and ELISA was performed, as previously reported (16). Briefly, deepfrozen specimens of $250-300 \mathrm{mg}$ wet weight: i) carcinoma cells only; ii) tumor cell-free stromal tissue; iii) not microdissected tumor tissue were pulverized by a microdismembrator (Satorius, Göttingen, Germany). The resulting powder was suspended in $1.8 \mathrm{ml}$ TBS $(0.002 \mathrm{M}$ Tris $\mathrm{HCl}$, $0.125 \mathrm{M} \mathrm{NaCl}, \mathrm{pH} 8.5)$ and $0.2 \mathrm{ml}$ of the nonionic detergent Triton X-100 10\% (Sigma, München, Germany) yielding a 1\% Triton X-100 final preparation. After gentle stirring for $12 \mathrm{~h}$ at $4^{\circ} \mathrm{C}$, the suspension was subjected to centrifugation $\left(21,000 \mathrm{x} \mathrm{g}\right.$ for $\left.60 \mathrm{~min}, 4^{\circ} \mathrm{C}\right)$ in order to separate cell debris. The total protein content of the extract was measured by using a conventional biuret-protein reaction assay (bca protein assay kit; Pierce, IL, USA). We performed an UPA, UPAR and PAI-1 ELISA using commercially available ELISA kits (American Diagnostica, Pfungstadt, Germany, Immubind \#893, \#894, \#821) and a conventional ELISA-reader (Thermo Multiscan EX) according the manufacturer's instructions. The measurements were performed in duplicates. UPA, uPAR and PAI-1 content was determined in the Triton X-100 extract and calculated per mg of tissue protein.

RNA extraction, reverse transcription $(R T)$. RNA was obtained from microdissected myoepithelial cells (1500-2000 cells in each case) with the Micro RNA isolation kit (Stratagene, Germany). The RNA pellet was redissolved in $15 \mu \mathrm{l}$ sterile DEPC-treated water and incubated with $1 \mu 1$ of RNase inhibitor (PE Applied Biosciences, Germany) and 20 units of DNase I (GenHunter, Germany) for $2 \mathrm{~h}$ at $37^{\circ} \mathrm{C}$ in a total volume of $20 \mu \mathrm{l}$. The amount and purity of RNA was calculated by using an Agilent Bioanalyser 2100. The RT reaction was carried out in a total volume of $40 \mu \mathrm{l}$ : $1 \mathrm{X} \mathrm{RT}$ buffer [500 $\mu \mathrm{M}$ deoxynucleotide triphosphates, $3 \mu \mathrm{M}$ random primers, 60 units of RNasin and 200 units of Superscript RNase $\mathrm{H}^{-}$(Invitrogen)]. To this mixture, we added $1 \mu \mathrm{g}$ of total RNA. The reaction was allowed to proceed for $60 \mathrm{~min}$ at $37^{\circ} \mathrm{C}$ followed by $5 \mathrm{~min}$ at $95^{\circ} \mathrm{C}$ and a subsequent rapid cooling on ice. The cDNA was strored at $-20^{\circ} \mathrm{C}$ until further use.

After re-extraction of RNA, reverse transcription was performed using $12 \mu \mathrm{l}$ of total RNA, $2.5 \mu \mathrm{M}$ random hexameres, $25 \mu \mathrm{M}$ dNTPs and 100 units of MMLV reverse transcriptase (Invitrogen, Germany). For each sample, a mock reaction without addition of reverse transcriptase was performed.

Qualitative and quantitative cDNA amplification. A measure of $1 \mu 1$ of the UPAR cDNA product was amplified in a thermal cycler (Autogene II Grant, Germany) for 35 cycles consisting of $60 \mathrm{sec}$ at $95^{\circ} \mathrm{C}, 90 \mathrm{sec}$ at $55^{\circ} \mathrm{C}$ and $3 \mathrm{~min}$ at $72^{\circ} \mathrm{C}$. Taq polymerase was obtained from Perkin-Elmer Cetus and used according to the supplier's instructions. The following primers were based on the published uPAR (17) and PAI-1 (18) sequence and synthesised by MWG Biotech, Germany: uPAR sense, 5'-CATGCAGTGTAAGACCAACG-3'; uPAR antisense, 5'-CTCTCACAGCTCATGTCTGATGAGCCAC-3'; 
Table I. uPA, uPAR and PAI-1 immunoreactions in 30 invasive breast carcinomas, 30 DCIS and 10 cases of normal (non-tumor) breast tissue.

\begin{tabular}{|c|c|c|c|c|c|}
\hline Antibody & uPAR IID7 & uPAR HU277 & uPAR 3936 & PAI-1 & uPA \\
\hline \multicolumn{6}{|l|}{ Breast cancer $(n=30)$} \\
\hline Tumor cells & $28(8 / 12 / 8)$ & $27(7 / 12 / 8)$ & $17(6 / 6 / 5)$ & $28(6 / 14 / 8)$ & $29(7 / 14 / 8)$ \\
\hline Stromal cells & 30 & 30 & 30 & 30 & 30 \\
\hline Endothelial cells & 28 & 20 & 0 & 30 & 30 \\
\hline \multicolumn{6}{|l|}{ DCIS $(n=30)$} \\
\hline Myoepithelial cells & $28(9 / 10 / 9)$ & $20(8 / 6 / 6)$ & $19(7 / 6 / 5)$ & $28(8 / 11 / 9)$ & $29(9 / 11 / 9)$ \\
\hline Tumor cells & $28(9 / 10 / 9)$ & $20(8 / 6 / 6)$ & $19(7 / 6 / 5)$ & $28(8 / 11 / 9)$ & $29(9 / 11 / 9)$ \\
\hline Stromal cells & 30 & 30 & 30 & 30 & 30 \\
\hline Endothelial cells & 28 & 18 & 0 & 30 & 28 \\
\hline \multicolumn{6}{|l|}{ Breast tissue $(n=10)$} \\
\hline Myoepithelial cells & 4 & 4 & 3 & 4 & 5 \\
\hline Epithelial cells & 6 & 5 & 4 & 4 & 5 \\
\hline Stromal cells & 5 & 4 & 3 & 5 & 5 \\
\hline Endothelial cells & 5 & 4 & 0 & 5 & 5 \\
\hline
\end{tabular}

uPA, urokinase type-plasminogen activator; PAI-1, plasminogen activator inhibitor type-1; uPAR, urokinase type-plasminogen activator receptor; DCIS, ductal carcinoma in situ; stromal cells, macrophages and fibroblasts. For anti-uPAR immunoreaction three different antibodies were used: mAb IID7, pAb HU277, mAb 3936; for anti-uPA and anti-PAI-1 immunoreaction mAb anti- uPA 3688 and anti-PAI-1 3785 were used. The WHO grading for DCIS was used (low grade $n=9$, intermediate grade $n=12$, high grade $n=9$ ). The invasive carcinomas were graded according to the Elston and Ellis criteria (grade $1 n=8$, grade $2 n=14$, grade $3 n=8$ ). Behind the number of positive cases the results of grades 1-3 (DCIS and invasive carcinomas) are given in parantheses.

PAI-1 sense, 5'-ACACCCTCAGCATGTTCATT-3'; and PAI-1 anti-sense, 5'-CTCGATCTTCACTTTCTGCA-3'.

The amplification products showed the expected size of 311 (uPAR) and 290 (PAI-1) base pairs. Appropriate negative controls including amplification of the mock $\mathrm{RT}$ reaction product were performed in each run. The polymerase chain reaction $(\mathrm{PCR})$ products were separated on a $2 \%$ agarose gel stained with ethidium bromide.

Real-time PCR was performed in a LightCycler instrument using LC-Fast start reaction mix SYBR-Green I (Roche Diagnostics). Polymerase chain reaction amplification was carried out in a final volume of $10 \mu 1$ containing $1 \mu 1$ of cDNA sample; $1.2 \mu 1 \mathrm{MgCl}_{2}(25 \mathrm{mM}) ; 0.2 \mu 1$ of PAI-1-primers (25 $\mu \mathrm{M}$ each); and $1 \mu \mathrm{l}$ LC FastStart DNA Master SYBRGreen I/Enzyme Mix (including Taq DNA polymerase, reaction buffer and deoxynucleotide triphosphate mixture). After an initial step of $10 \mathrm{~min}$ at $95^{\circ} \mathrm{C}$ (cDNA denaturation/ HotStart-Taq polymerase activation), 40 amplification cycles were performed: $15 \mathrm{sec}$ at $95^{\circ} \mathrm{C}, 5 \mathrm{sec}$ at $58^{\circ} \mathrm{C}$ and $15 \mathrm{sec}$ at $72^{\circ} \mathrm{C}$.

After PCR, a melting curve was created by increasing the temperature from 61 to $99^{\circ} \mathrm{C}$ with a temperature transition rate of $0.1^{\circ} \mathrm{C} \mathrm{sec}-1$. Each PCR experiment was performed in triplicate. For every LightCycler run, a standard curve was generated by the detection of the crossing point $(\mathrm{CP})$ of each standard. The concentrations of unknown samples were then calculated by comparing their CPs to the standard curve.

Statistical analysis. Results are expressed as the mean \pm standard error of the mean (SEM) and are considered significant at the $\mathrm{p}<0.05$ level (two-tailed). Wilcoxon MannWhitney (rank sum test) was used for statistical analyses.

\section{Results}

Immunohistochemistry and in situ hybridization. To localize UPA, UPAR and PAI-1, we have studied 30 cases of invasive breast carcinomas, 30 cases of DCIS and 10 cases of normal (benign, non-tumor) breast tissue for expression and synthesis of these factors by imunohistochemistry and in situ hybridization. The results of immunohistochemistry are listed in Table I. Remarkably in all cases analyzed stromal cells (macrophages and fibroblasts) of invasive carcinomas and DCIS were uPA-, uPAR- and PAI-1-positive. In only $50 \%$ of normal (non-tumor) breast tissue stromal cells showed an anti-uPA, anti-uPAR and anti-PAI-1 immunoreaction. In these cases, fibroblasts and macrophages were only occasionally positive. All cases were probed for the presence of UPAR and PAI-1 mRNA by in situ hybridization using antisense oligodeoxynucleotides. With no exception macrophages, fibroblasts, tumor cells (DCIS and invasive carcinomas), epithelial cells (of normal breast tissue) and myoepithelial cells as well as endothelial cells showed a positive reaction with the antisense probe. No reactions at all were seen with the sense probes (Figs. 1 and 2).

Separating tumor stroma and cancer cells by laser capture microdissection followed by ELISA analysis. This is the first study in which tumor cells and stromal tissue of invasive breast carcinomas were separated by laser capture 

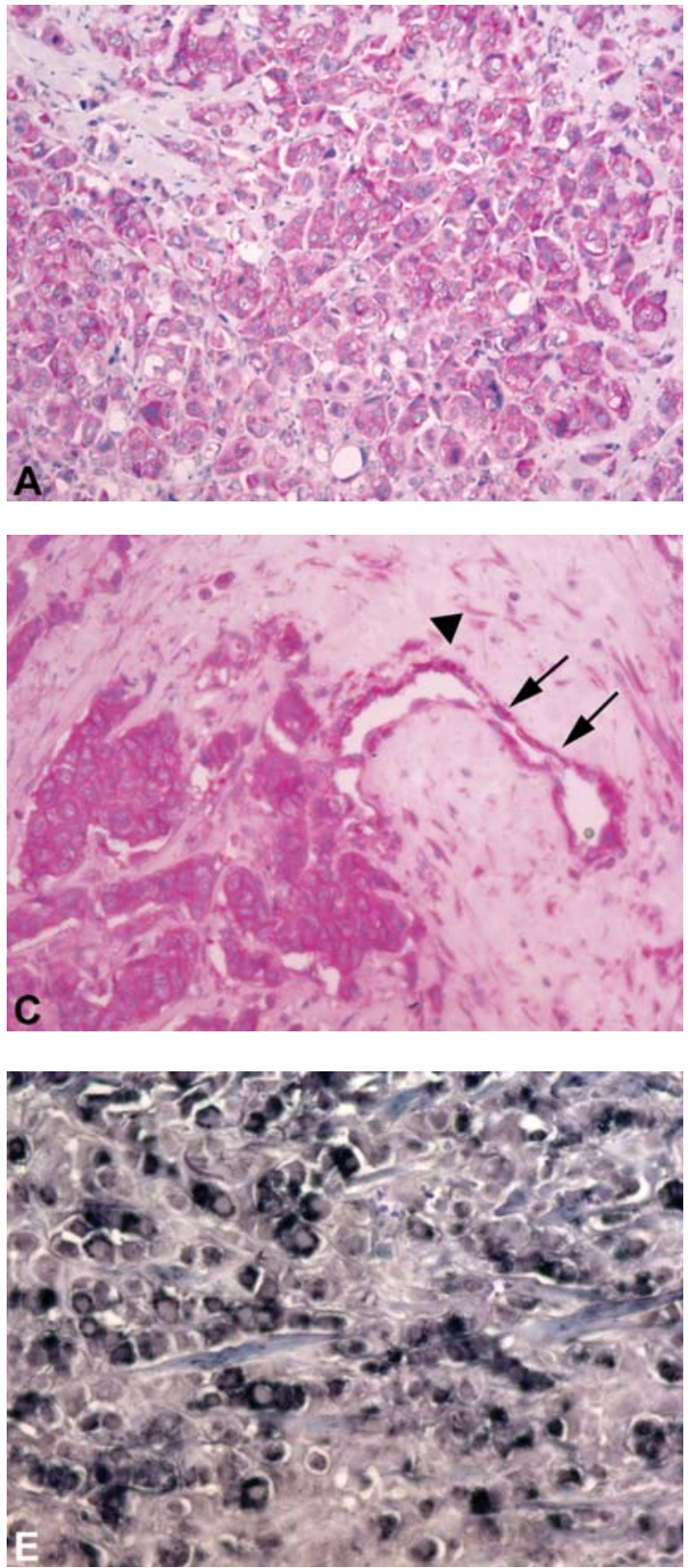
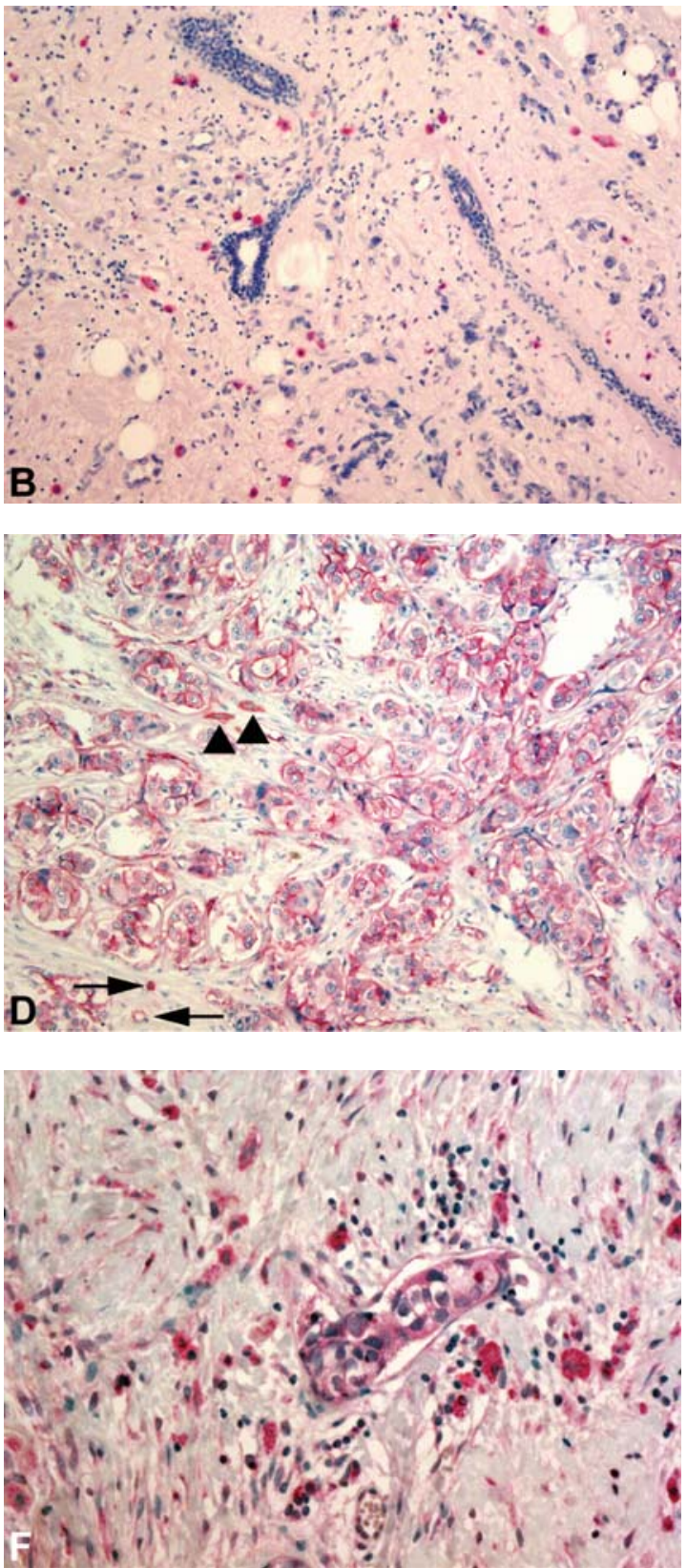

Figure 1. (A) Anti-uPA immunoreaction of an invasive ductal carcinoma. Tumor cells show strong staining. (B) Anti-uPA immunoreaction of an invasive lobular carcinoma. Tumor-associated macrophages show strong immunoreaction. Tumor cells and normal epithelial cells are negative. (C) Anti-PAI-1 immunoreaction of an invasive ductal carcinoma. Tumor cells, endothelial cells (arrows) and stromal cells (arrowhead) are positive. (D) Anti-uPAR HU277 of an invasive ductal carcinoma. Tumor cells, stromal cells (macrophages and fibroblasts) (arrowheads) as well as endothelial cells (arrow) exhibit the uPAR antigen. (E) In situ hybridization for uPAR mRNA in invasive ductal breast carcinoma. Tumor cells are positive (black). (F) Lymphangio-invasion within a desmoplastic stroma reaction of an invasive ductal carcinoma showing a strong anti-PAI-1 immunoreaction of fibroblasts, macrophages, endothelial cells and intravascular tumor cells.

microdissection followed by ELISA-based determination of the UPA, UPAR and PAI-1 levels. When tumor cells and stroma were not separated by laser microdissection the mean $( \pm$ SEM) uPA level $(\mathrm{n}=30)$ was $2.83 \pm 0.63 \mathrm{ng} / \mathrm{mg}$ (range $0.04-13.2 \mathrm{ng} / \mathrm{mg})$, the mean $( \pm$ SEM) uPAR level $(\mathrm{n}=30)$ was $4.31 \pm 0.32 \mathrm{ng} / \mathrm{mg}$ (range $0.03-13.5 \mathrm{ng} / \mathrm{mg}$ ) and the mean ( \pm SEM) PAI-1 level $(\mathrm{n}=30)$ was $14.2 \pm 3.1 \mathrm{ng} / \mathrm{mg}$ (range 2.4-38.4 ng/mg). When tumor cells and stroma were separated by laser microdissection the mean ( \pm SEM) uPA level of tumor cells only $(\mathrm{n}=30)$ was $2.89 \pm 0.53 \mathrm{ng} / \mathrm{mg}$ (range $0.03-11.3 \mathrm{ng} / \mathrm{mg}$ ), the mean $( \pm$ SEM) uPAR level of tumor cells only $(\mathrm{n}=30)$ was $4.02 \pm 0.41 \mathrm{ng} / \mathrm{mg}$ (range
0.04-12.5 ng/mg) and the mean ( \pm SEM) PAI-1 level of tumor cells only $(\mathrm{n}=30)$ was $13.2 \pm 3.1 \mathrm{ng} / \mathrm{mg}$ (range $0.68-39.1 \mathrm{ng} / \mathrm{mg}$ ). When tumor cells and stroma were separated by laser microdissection the mean ( \pm SEM) uPA level of stromal cells only $(\mathrm{n}=30)$ was $3.22 \pm 0.7 \mathrm{ng} / \mathrm{mg}$ (range $0.02-11.7 \mathrm{ng} / \mathrm{mg}$ ), the mean $( \pm$ SEM) uPAR level of stromal cells only $(n=30)$ was $4.34 \pm 0.44 \mathrm{ng} / \mathrm{mg}$ (range $0.04-13.3 \mathrm{ng} / \mathrm{mg}$ ) and the mean ( \pm SEM) PAI-1 level of stromal cells only $(n=30)$ was $14.3 \pm 3.1 \mathrm{ng} / \mathrm{mg}$ (range $0.84-40.2 \mathrm{ng} / \mathrm{mg}$ ). The uPA, uPAR and PAI-1 level of not separated tumor tissue, tumor cells only and stromal tissue only did not show any significant differences in statistical analysis ( $\mathrm{p}>0.05)$. 

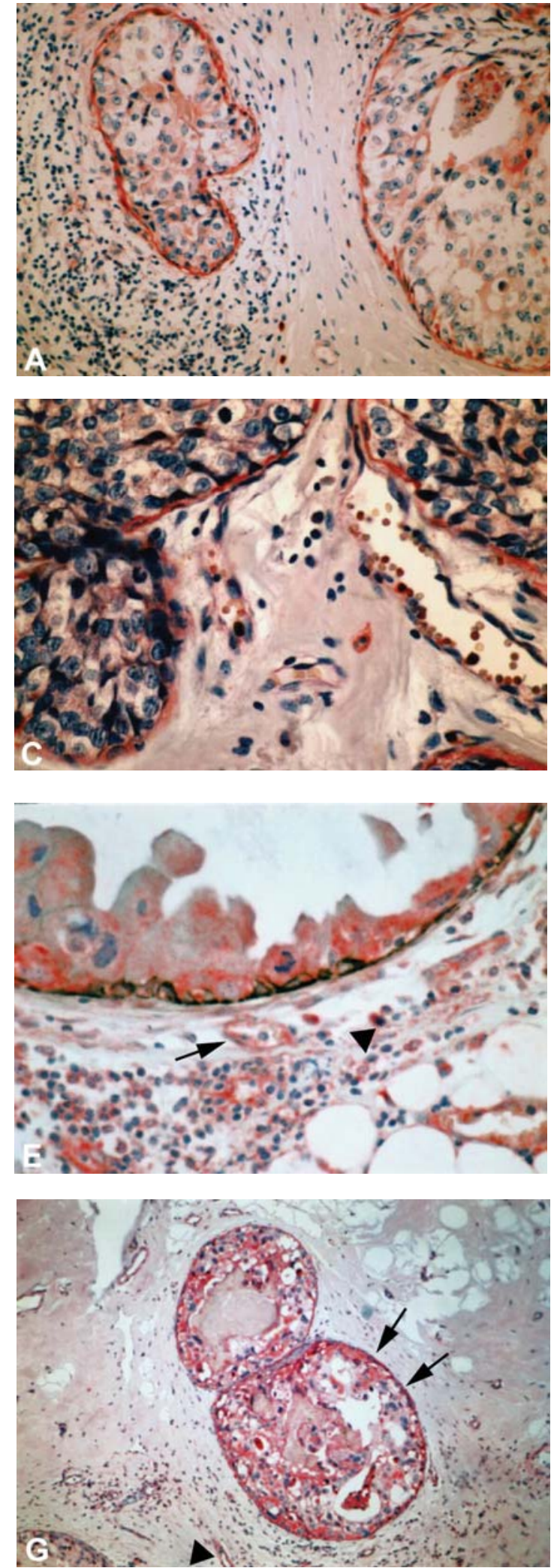
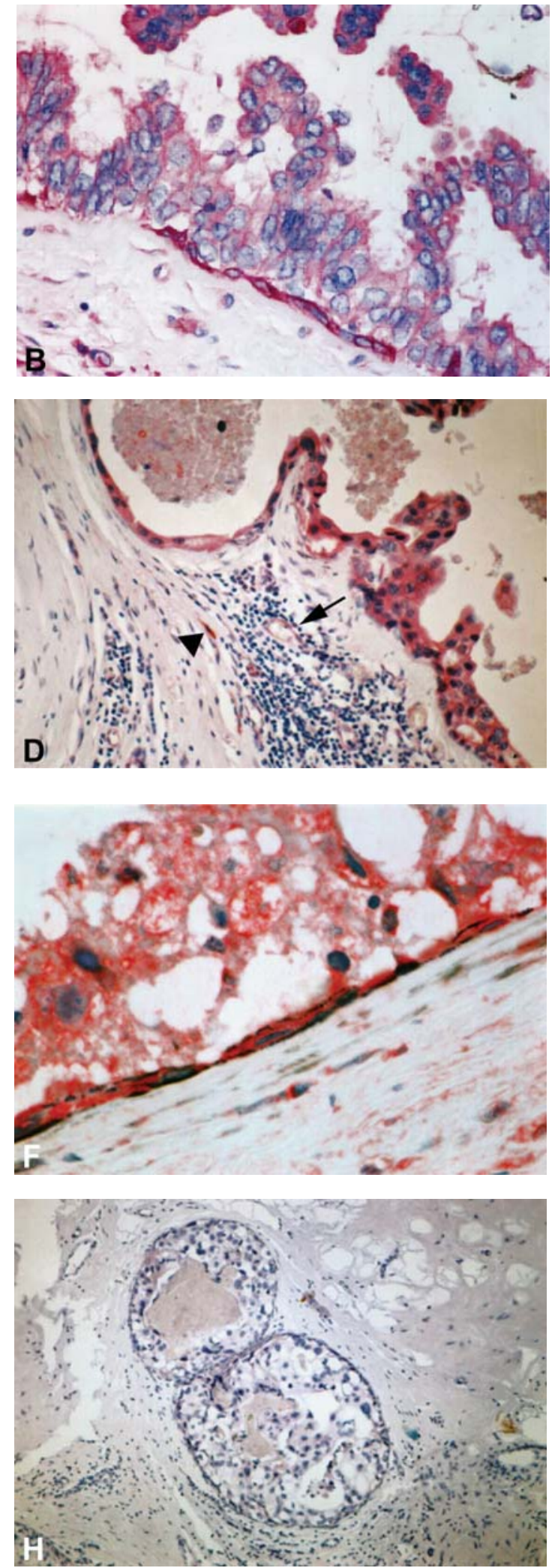

Figure 2. (A) High-grade DCIS stained with pAb anti-uPAR HU277. Myoepithelial cells and macrophages show a strong positive anti-uPAR immunoreaction. Tumor cells show only a faint reaction. (B) Intermediate grade DCIS showing an incomplete myoepithelial cell layer with a strong anti-PAI-1 immunoreaction; tumor cells show faint immunoreaction. (C) Same DCIS as in image A stained with mAb anti-uPAR IID7; the myoepithelial cell layer and macrophages exhibit the uPAR antigen; tumor cells and endothelial cells are negative. (D) Anti-PAI-1 immunoreaction of a high-grade DCIS. Tumor cells, endothelial cells (arrow) and macrophages as well as fibroblasts (arrowhead) are positive. The myoepithelial cell layer is absent; in corresponding tissue sections no myoepithelial cells in this duct were observed using anti-calponin and anti-SMA antibodies. (E) Represents a high-grade DCIS double-stained for anti-PAI-1 (red) and anti-smooth muscle actin (SMA) (black); myoepithelial cells are positive for both anti-PAI-1 and anti-SMA; tumor cells, stromal cells (arrowhead) and endothelial cells (arrow) strongly express the PAI-1 protein. (F) Represents a high-grade DCIS double-stained for anti-uPAR IID7 (red) and anti-smooth muscle actin (SMA) (black); myoepithelial cells are positive for both anti-uPAR and anti-SMA; tumor cells and stromal cells strongly express the uPAR antigen. (G and $\mathrm{H}$ ) Non-isotopic in situ hybridization using fluorescein-labeled oligodeoxynucleotides complementary to PAI-1 mRNA in a non-high-grade DCIS with necrosis; (G) antisense-probe, a distinct reaction in myoepithelial cells (arrows), tumor cells, stromal cells and endothelial cells (arrowhead) is observed; (H) no reaction is seen with the sense-probe. 

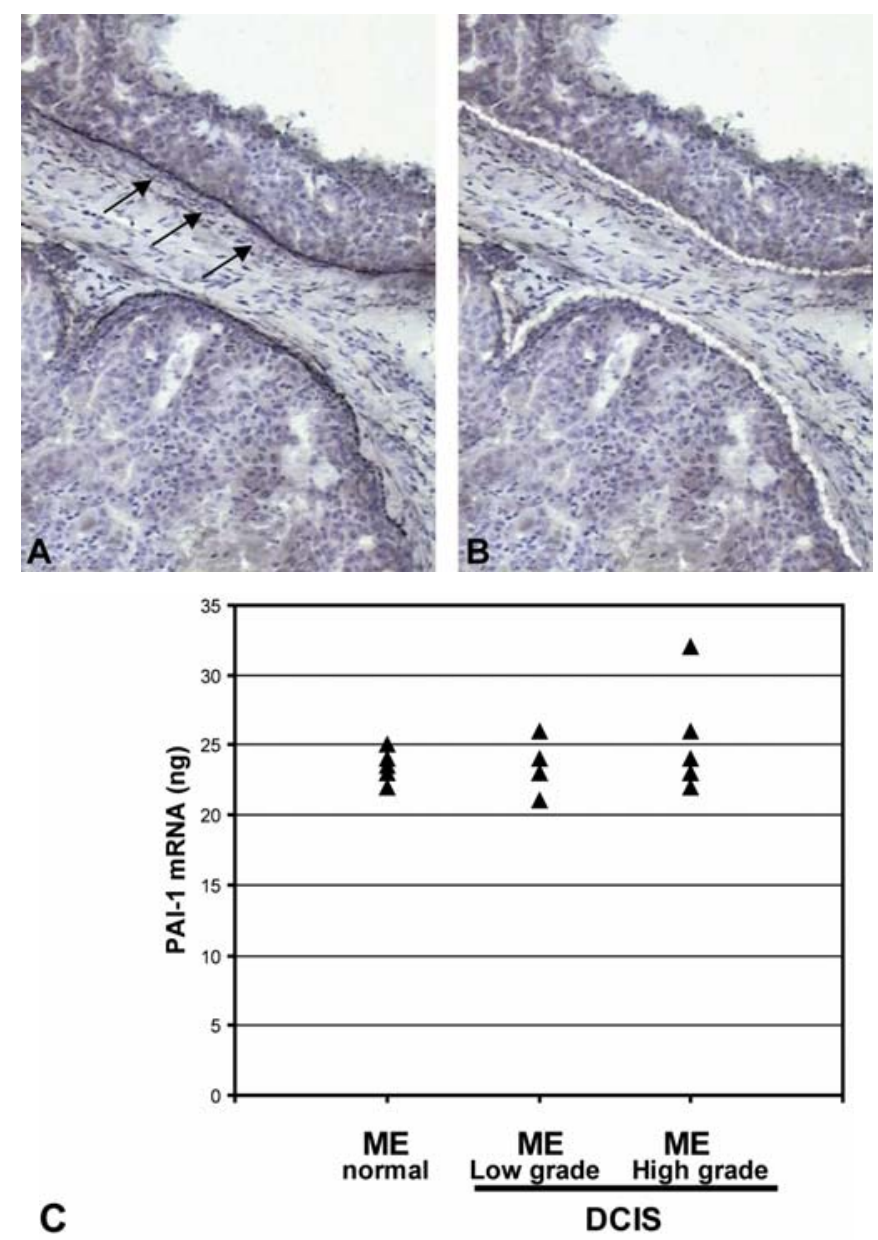

C

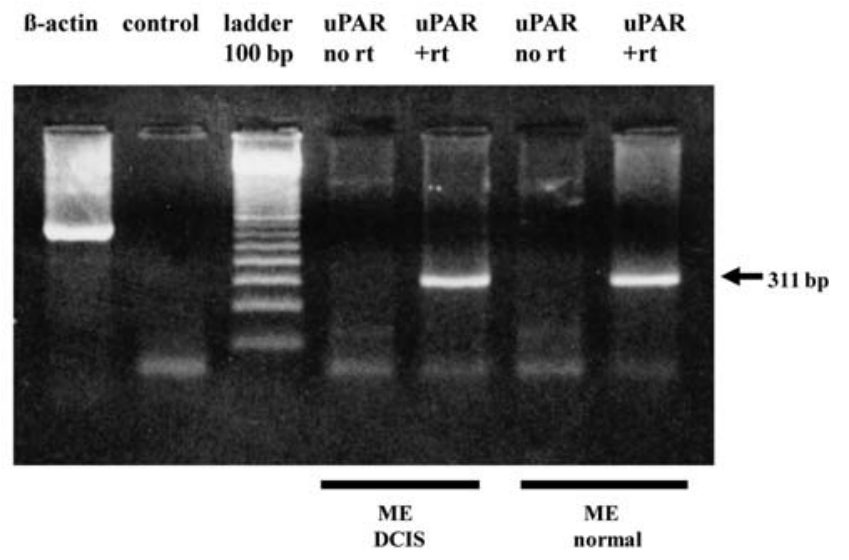

D

Figure 3. (A) Frozen section of a high-grade DCIS stained with mAb antismooth muscle actin. Myoepithelial cells (arrows) show black staining. (B) Myoepithelial cells were captured by laser-microdissection. (C) Quantitative rt-PCR of mRNA derived from microdissected myoepithelial cells (ME) of normal breast tissue, low-grade DCIS and high-grade DCIS using PAI-1specific primers. Five cases of each group were selected and a LightCycleranalysis was performed. (D) The RNA of myoepithelial cells (DCIS and normal breast tissue) was isolated, reverse-transcriptase reaction followed by PCR using uPAR primers (see methods) was performed. The RT-PCR reveals a 311 bp product in both probes (DCIS and normal breast tissue); without RT-reaction no product was received.

Myoepithelial cells. Prompted by our immunohistological and in situ hybridization results that nearly all myoepithelial cells exhibit UPA, uPAR and PAI-1, we investigated these important host cells in detail. In double immunostaining analysis we have demonstrated, that myoepithelial cells exhibit UPAR and PAI-1 (Fig. 2E and F). Additionally, we isolated myoepithelial cells from normal breast tissue and from DCIS by laser capture microdissection followed by PCR using specific primers for UPAR and PAI-1 mRNA. The amplification products showed the expected size of 311 (uPAR) and 290 (PAI-1) base pairs. Results are demonstrated in Fig. 3. Furthermore, we have demonstrated by immunofluorescence double staining that the extracellular matrix protein vitronection and collagen type- 4 are colocalised in the basement membrane of DCIS and normal breast ducts/acini. In Fig. 4, one can see that both proteins are associated within the basement membrane of a DCIS and vessel walls.

\section{Discussion}

Factors of the plasminogen activator system play a key role in tumor invasion and metastasis of breast cancer. In particular, breast cancer patients can be identified by antigen determination (ELISA) of these factors in tumor tissue extracts allowing a more individualized delineation of patients to be treated by adjuvant therapy (7). uPA and PAI-1 were the first novel tumor biological factors to be validated at the highest level of evidence (LOE Ia) regarding their clinical utility in breast cancer (4). However, it is unclear whether it is their (relative) levels in the tumor stroma or in the tumor cells themselves that is most relevant to patients outcome. This missing knowledge leads to an uncertainty concerning managing the breast cancer tissue specimens. It is unclear how much tumor stroma is allowed in one tumor tissue specimen for using this method correctly for a valid prediction of the individual patient's outcome. We have demonstrated that no significant differences between UPA, uPAR and PAI-1 levels determined by ELISA in tumor stroma only, tumor cells only and not separated breast cancer tissue exist $(p>0.05)$. Our results suggest that similar expression levels of these factors in tumor stroma only, tumor cells only and in not separated breast cancer tissue may have the same impact on the clinical behaviour of breast cancer. These results are an important issue for practical use of tissue sampling. For using uPA and PAI-1 levels as prognostic and predictive factors in breast cancer the quantity of tumor stroma in the tumor tissue specimen is not relevant for assessment patients outcome. This conclusion was confirmed by our immunohistochemistry and in situ hybridization results showing that in nearly all cases of invasive carcinomas and DCIS fibroblasts as well as macrophages strongly express uPA, uPAR and PAI- 1 . Interestingly, in only $50 \%$ of normal breast tissue these factors are expressed by non-tumor-associated stromal cells. In contrast to most of the other studies, we found a strong expression of the uPA-system in tumor-associated macrophages and fibroblasts in all invasive breast carcinomas and in DCIS. In the present study, we furnish evidence that tumor-associated macrophages (TAM) and tumoral fibroblasts strongly express uPA, uPAR and PAI-1 in all cases examined. In normal (non-neoplastic) breast tissue fibroblasts express UPA, uPAR and PAI-1 in only 50\%. The increased expression (ELISA and immunohistochemistry) in TAMs and tumoral fibroblasts implies that there is a specific response to tumor 

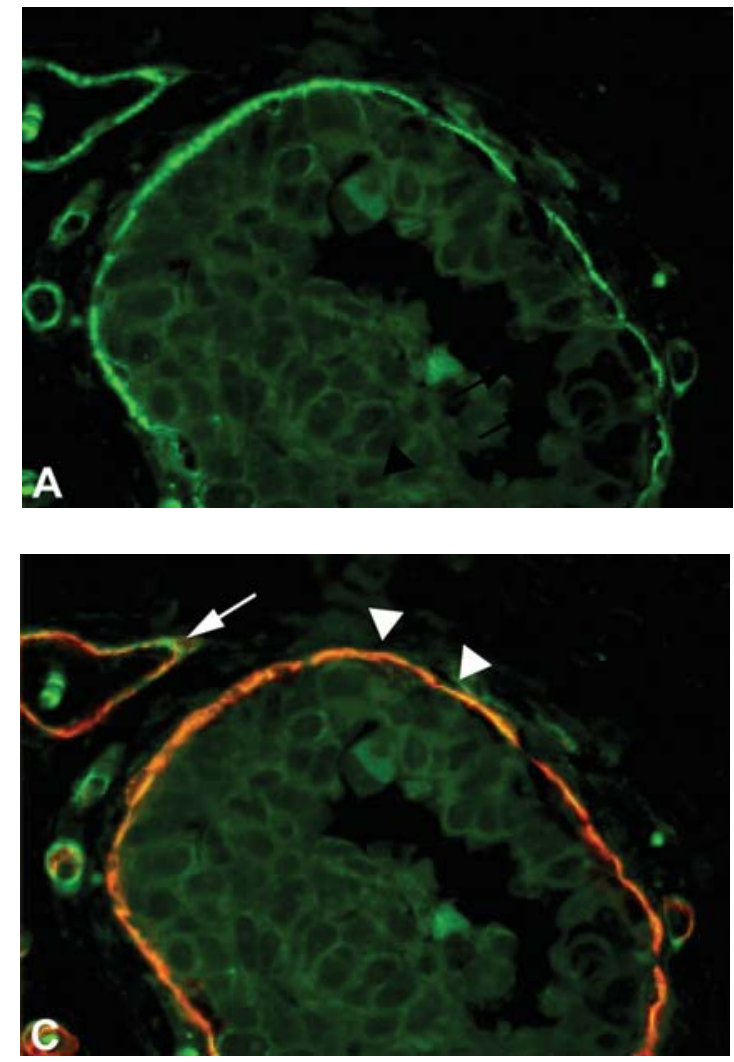
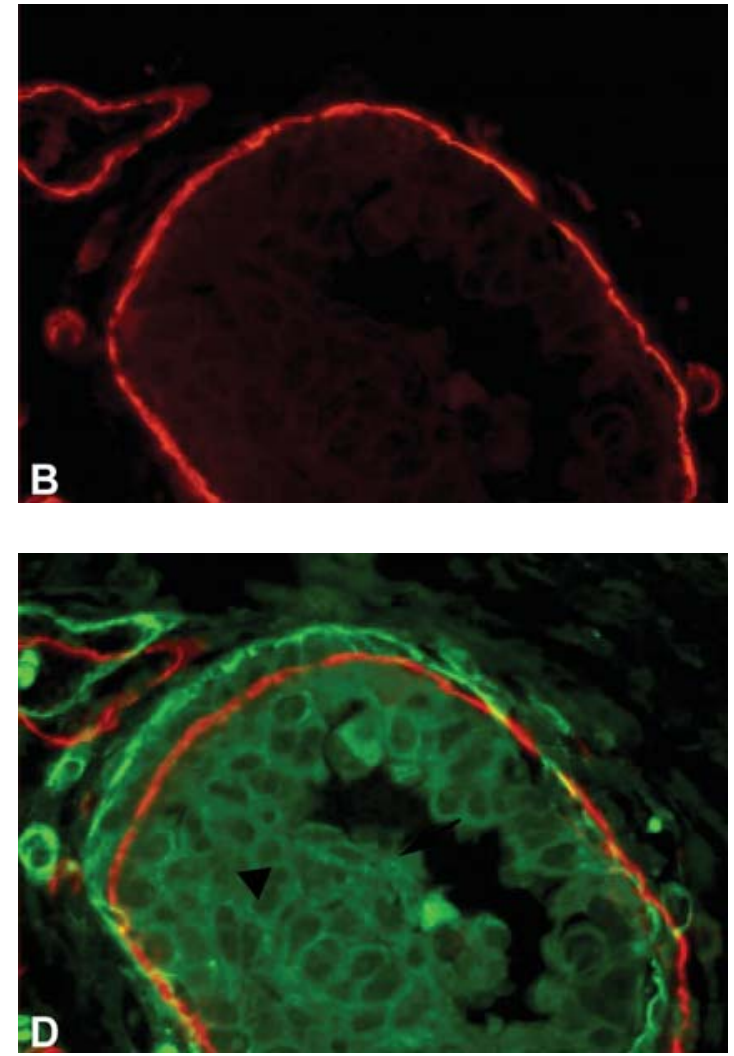

Figure 4. Immunofluorescence double staining of a high-grade DCIS with mAb anti-vitronectin (A, red signal) and anti-collagen type-4 (B, green signal); (C) both immunoreactions are associated within the basement membrane of the breast duct (arrowheads) and of a blood vessel (arrow); (D) DCIS double immunolabelled for vitronectin and collagen type-4 with a 10-pixel shift of the red signal (collagen type-4), revealing clearly a green and red signal in the basement membranes.

growth. The lower levels found in macrophages and fibroblasts of normal breast tissue also indicate that there is more to the tumor stroma response than is suggested by the concept that tumors are wounds that do not heal (19).

Prompted by our immunohistological results (nearly all myoepithelial cells exhibit uPA, uPAR and PAI-1), we investigated the myoepithelial cells in detail. The myoepithelial cells of the breast ducts and breast glands may play a special role concerning the invasion of tumor cells because of their important anatomic location between the basement membrane and the epithelial cells/non-invasive tumor cells of DCIS. The myoepithelial cell, which lies on the epithelial side of the basement membrane, is thought to contribute considerably to both the synthesis and remodeling of this structure. The myoepithelial cell also lies in direct juxtaposition to normally proliferating and differentiating cells in healthy breast tissue and to abnormally proliferating and differentiating epithelial cells in precancerous lesions of the breast. In this study, we have demonstrated by multimodal methods that UPAR and PAI-1 protein and mRNA is expressed in most myoepithelial cells of the normal duct and lobular system and in DCIS. This is an important issue to address since the uPA-system plays an important role in matrix degradation and invasion.

In addition to promoting cell migration by focusing uPA proteolytic activity to the cell surface, uPAR can physically be associated with another ligand, vitronectin, mediating cell adherence to the extracellular matrix such as basement membrane. We have demonstrated by double-staining that the extracellular matrix protein vitronectin $(\mathrm{Vn})$ and collagen type 4 are co-localized in the basement membrane of DCIS and normal breast ducts/acini. In Fig. 4 (double staining of a DCIS using anti-collagen type-4 and anti-vitronectin mAb), one can see that both proteins are associated within the basement membrane of a breast duct and vessel walls. This is not surprising since a strong interaction of $\mathrm{Vn}$ and collagen type-4 in vitro is well known (20). Furthermore, a colocalization of both proteins is known in the basement membrane of the kidney tubulus and of vascular basement membranes $(21,22)$. The presence of $\mathrm{Vn}$ within the basement membrane and the expression of UPAR in myoepithelial cells of both DCIS and normal breast glands suggests an important cell-matrix interaction which regulates the cell adhesion and detachment. uPA is the physiological activator of this 'vitronectin' receptor, which means that uPA stabilizes the vitronectin-uPAR binding and thereby the cell-matrix contact. PAI-1 is not only a protease inhibitor, PAI-1 resolves the vitronectin-uPAR binding and releases the cells from the cell-matrix contact (23). Therefore, UPAR in myoepithelial cells of the breast may play a multifunctional role. In the normal breast tissue, uPAR is necessary for the physiological shedding of epithelial and myoepithelial cells. By focusing the proteolytic enzyme uPA on the cell surface, uPAR of myoepithelial cells take part in the remodeling of the basement membrane.

In two cases of DCIS, a few ducts showed an incomplete myoepithelial cell layer expressing uPAR and PAI-1 (Fig. 2B and D). In these cases, a few ducts had an incomplete/complete loss of the myoepithelial cell layer. A moderate anti-uPA 
immunoreaction of myoepithelial cells was found. In these interesting cases, we hypothesize that high levels of PAI-1 and low levels of uPA in myoepithelial cells are involved in the vitronectin/uPAR-mediated detachment of the myoepithelial cells. Sternlicht and coworkers (24) reported that myoepithelial cells regulate the progression of DCIS to invasive cancer by inhibiting cell invasion. They suggest that PAI-1 does not contribute to the anti-invasive phenotype of myoepithelial cells or, conversely, to a highly invasive and metastatic phenotype of tumor cells. Previously, we have demonstrated that PAI-1 may detach myoepithelial cells from basement membrane (HMS-4X, myoepithelial cell-line) in vitro (25). PAI-1 may also be an important component in detachment of myoepithelial cells in vivo, since PAI-1 is able to attenuated the cell-matrix interaction by resolving the uPAR/Vnbinding. We speculate that the loss of the anti-invasive myoepithelial cell-layer in DCIS may be triggered by PAI-1 and could be an early sign of subsequent invasion.

The predominant expression of components of the uPAsystem in tumor stroma and host bystander cells (e.g. myoepithelial cells) reflects the importance and hints at the collaboration of the stroma and cancer cells. A better understanding of stromal contributions to cancer progression will likely increase our awareness of the importance of the combinatorial signals that support and promote tumor growth, invasion and metastasis and eventually result in the identification of new therapeutics targeting the tumor stroma. Several strategies involving direct targeting of the uPA-system with other components such as integrins or fibronectin and also an inhibition of molecular transcriptional regulators of gene expression, should be anticipated as promising strategies to improve prognosis of cancer patients.

\section{Acknowledgements}

This study was supported by the Deutsche Krebshilfe Dr Mildred Scheel Stiftung für Krebsforschung, Germany, $10-1507 \mathrm{H}$ and by a grant from the Faculty of Clinical Medicine, Mannheim, University of Heidelberg. The authors gratefully appreciate the excellent technical assistance of Dr N. Arens and Dr G. Wolf.

\section{References}

1. Bishop JM: Molecular themes in oncogenesis. Cell 64: 235-248, 1991.

2. Hildenbrand R, Gandhari M, Stroebel P, Marx A, Allgayer H and Arens N: The urokinase-system-role of cell proliferation and apoptosis. Histol Histopathol 23: 227-236, 2008.

3. Binder BR, Mihaly J and Prager GW: uPAR-uPA-PAI-1 interactions and signaling: A vascular biologist's view. Thromb Haemost 97: 336-342, 2007.

4. Harbeck N, Kates RE, Gauger K, Willems A, Kiechle M, Magdolen V and Schmitt M: Urokinase-type plasminogen (UPA) and its inhibitor PAI-1: novel tumor-derived factors with high prognostic and predictive impact in breast cancer. Thomb Haemost 91: 450-456, 2004.

5. Harbeck N, Kates RE, Look MP, Meijer-van Gelder ME, Klijn JGM, Krüger A, Kiechle M, Jänicke F, Schmitt M and Foekens JA: Enhanced benefit from adjuvant chemotherapy in breast cancer patients classified high-risk according to urokinasetype plasminogen activator (uPA) and plasminogen activator inhibitor type-1. Cancer Res 62: 4617-4622, 2002.
6. Andreasen PA, Egelund R and Petersen HH: The plasminogen activator system in tumor growth, invasion, and metastasis. Cell Mol Life Sci 57: 25-40, 2000.

7. Schmitt M, Harbeck N, Thomssen C, Wilhelm O, Magdolen V, Reuning U, Ulm K, Höfler H, Jänicke F and Graeff H: Clinical impact of the plasminogen activation system in tumor invasion and metastasis: prognostic relevance and target for therapy. Thromb Heamost 78: 285-296, 1997.

8. Liotta LA, Steeg PS and Stetler-Stevenson WG: Cancer metastasis and angiogenesis: an imbalance of positive and negative regulation. Cell 64: 327-336, 1991.

9. Folkman J and Klagsbrun M: Angiogenic factors. Science (Washington DC) 235: 442-447, 1987.

10. Elston CW and Ellis IO: Pathological prognostic factor in breast cancer. 1. The value of histological grade in breast cancer: experience from a large study with long term follow-up. Histopathology 19: 403-410, 1991.

11. Luther T, Magdolen V, Albrecht S, Kasper M, Riemer C, Kessler H, Gaeff H, Müller M and Schmitt M: Epitope-mapped monoclonal antibodies as tools for functional and morphological analysis of human urokinase receptor in tumor tissue. Am J Pathol 150: 1231-1244, 1997.

12. Magdolen V, Rettenberger P, Lopens A, Oi H, Lottspeich F, Kellermann J, Creutzburg S, Goretzki L, Weidle U, Wilhelm O, Schmitt $\mathrm{M}$ and Graeff $\mathrm{H}$ : Expression of the human uPA receptor (uPA-R) in E. coli and CHO cells: purification of the recombinant proteins and generation of polyclonal antibodies in chicken. Electrophoresis 16: 813-816, 1995.

13. Hildenbrand R, Leitz M, Magdolen V, Luther T, Albrecht S, Graeff H, Stutte HJ, Bleyl UA and Schmitt M: Validation of immunolocalization of the urokinase receptor expression in ductal carcinoma in situ of the breast: comparison with detection by non-isotopic in-situ hybridization. Histopathology 36: 499-504, 2000.

14. Hildenbrand R, Glienke W, Magdolen V, Graeff H, Stutte HJ and Schmitt M: Urokinase receptor localization in breast cancer and benign lesions assessed by in situ hybridization and immunohistochemistry. Histochem Cell Biol 110: 27-32, 1998.

15. Fend F, Emmert-Buck MR, Chuaqui R, Cole K, Lee J, Liotta LA and Raffeld M: Immuno-LCM: Laser capture microdissection of immunostained frozen sections for mRNA analysis. Am J Pathol 154: 61-66, 1999

16. Hildenbrand R, Dilger I, Hörlin A and Stutte HJ: Urokinase and macrophages in tumour angiogenesis. Br J Cancer 72: 818-823, 1995.

17. Casey JR, Petranka JG, Kottra J, Fleenor DE and Rosse WF: The structure of the urokinase-type plasminogen activator receptor-gene. Blood 84: 1151-1156, 1994.

18. Ginsburg D, Zeheb R, Yang AY, Rafferty UM, Andreasen PA, Nielsen L, Dano K, Lebo RV and Gelehrter TD: cDNA cloning of human plasminogen activator-inhibitor from endothelial cells. J Clin Invest 78: 1673-1680, 1986.

19. Dvorak HF: Tumors: Wounds that do not heal. Similarities between tumor stroma generation and wound healing. N Engl J Med 315: 1650-1659, 1986.

20. Gebb C, Haymann EG, Engvall E and Ruoslahti E: Interaction with vitronectin and collagen. J Biol Chem 261: 16698-16703, 1986.

21. Falk RJ, Podack E, Dalmasso AP and Jennette JC: Localization of $\mathrm{S}$ protein and its relationship to the membrane attack complex of complement in renal tissue. Am J Pathol 127: 182190,1987

22. Sawa H, Sobel BE and Fuji S: Potentiation by hypercholesterolemia of the induction of aortic intramural synthesis of plasminogen activator inhibitor type-1 by endothelial injury. Circ Res 73: 671-680, 1993.

23. Wei Y, Lukashev M, Simon DI, Bodary SC, Rosenberg S, Doyle MV and Chapman HA Regulation of integrin function by the urokinase receptor. Science 273: 1551-1555, 1996.

24. Sternlicht MD, Kedeshian P, Shao ZM, Safarians S and Barsky SH: The human myoepithelial cell is a natural tumor suppressor. Clin Cancer Res 3: 1949-1958, 1997.

25. Hildenbrand R: The urokinase system in breast cancer and its precursor lesions. Recent Res Devel, Histopathol 1, Res Signpost, Kerala, pp1-19, 2002. 